\title{
Hydraulic networks with pressure-dependent closure relations, under restrictions on the value of nodal pressures. Maxwell matrix properties and monotonicity of flow distribution problem solution
}

\author{
Leonid Korelstein ${ }^{1, *}$ \\ ${ }^{1}$ PSRE Co, Moscow, Russia
}

\begin{abstract}
In the article, which continues the research of article [1], the results of previous article are generalized to "abstract" hydraulic networks. Additional existence theorems are proved for classical flow distribution problem (CFDP) for hydraulic networks with pressure-dependent closure relations, under restriction on nodal pressures. Hydraulic network Maxwell matrix properties are establish, related to monotonicity of CFDP solution.
\end{abstract}

This article is a continuation of article [1] and uses the same notations.

\section{Existence of CFDP solution for restricted nodal pressures}

A general CFDP solution existence theorem is proved in [0]. But for practical purposes it is desirable to establish condition of CFDP solvability for hydraulic network with limited definition region of closure relations. To some extend this is posible.

First we will consider simple (of often used and important) special case.

We will can network passive, if all its edges are passive. Special CFDP case, when all $Q_{f i x}=0$, we will call capacity calculation problem (CCP).

Theorem 1 (CCP solution existence for passive networks)

Let $G$ - connected graph of passive hydraulic network with edges closure relations defined on $\Omega \times \Omega$ (where $\Omega \subseteq \mathbb{R}$ - some non-empty open connected set), which fit strict monotonicity and continuity conditions. Consider CCP on $G$ with $Q_{f i x}=0$ and $P_{f i x} \in \Omega^{N_{P}}$. Then the solution of CCP exists, and for it $P_{v a r} \in\left[\min \left(P_{f i x}\right), \max \left(P_{f i x}\right)\right]^{N_{Q}}$.

Proof of theorem 1.

The theorem directly follows from theorem 4 [0] on CFDP solution existence for intermediate source data, if we will take zero set inflows and set nodal pressures equal to $\min \left(P_{f i x}\right)$ or $\max \left(P_{f i x}\right)$, as boundary CFDP source data.

\footnotetext{
* Corresponding author: korelstein@truboprovod.ru
} 
Is it possible to extend this theorem to general case with non-zero inflows and active edge network? Is it possible, for example, to estimate existence of CFDP solution on the base of direct calculations along simple paths in non-directed graph $\bar{G}$, which corresponds $G$ ? It is found out to be possible!

Consider simple paths in $\bar{G}$. We will call them P-paths, if first node $\in V_{P}$, and all others $\in V_{Q}$. Let $P a-$ P-path. We can change edge direction if necessary, so they all would directed along the path. We will tell that $P a$ «passes» flow $X$, if CFDP on path $P a$ with set pressure in start node, inflow $-X$ in end node and zero inflows in intermediate nodes (if any) is solvable. It is evident, that in order to determine if the path passes the flow, it is enough to simply directly calculate its edges one by one along the path - i.e. if the value $f_{L}\left(P_{F}, \mathrm{X}\right)$ is defined for each edge, and if this pressure $\in \Omega$. Note by $f_{L_{-} P a}\left(P a, P_{F}(P a), \mathrm{X}\right)$ pressure in end path depending on start node pressure and flow rate. If closure relations are continuous and strictly monotonic, this function for any path is also continuous, monotonically increase by start node pressure and monotonically decrease by flow rate. Also it is evident, that if path passes flows $X_{1}$ and $X_{2}, X_{1} \leq X_{2}$, then it passes also any intermediate flow $X\left(X_{1} \leq X \leq X_{2}\right)$. Moreover, if we will set any intermediate flow rates on path's edges (between $X_{1}$ and $X_{2}$ ), maybe not all equal each other, then direct calculation along the path will produce nodal pressures between values of nodal pressure for flows $X_{2}$ and $X_{1}$, which will $\in \Omega$. Further we will drop second parameter for simplicity and write end pressure simply as $f_{L_{-} P a}(\mathrm{~Pa}, \mathrm{X})$.

For any P-component we can define values of summary positive and negative set inflows: $Q^{+}=\sum_{v, Q_{f i x}(v)>0} Q_{f i x}(v), Q^{-}=\sum_{v, Q_{f i x}(v)<0} Q_{f i x}(v)$, where summation is on all nodes of P-component with set positive (negative) inflows. If there are no such nodes, $Q^{+}$ or $Q^{-}$are set to 0 . It is evident that $Q^{+} \geq 0 \geq Q^{-}$. Note that some flow rates in CFDP solution can be significantly greater than these values (thank to flow between node from $\in V_{P}$, and/or flow circulation).

The following (intuitively expected) theorem is valid.

Theorem 2 (On CFDP existence for restricted pressures).

Let $G$ - connected graph with closure relations, defined on $\Omega \times \Omega$, and strictly monotonic and continuous, and some CFDP is defined on it. If all P-paths of graph pass flows $Q^{-}$and $Q^{+}$(of their P-components), the CFDP solution exists. The nodal pressure of CFDP solution in any node $u \in V_{Q}$ fit the following inequalities (in which maximum and minimum are defined by all P-paths, ending in $u$ )

$$
P^{-}(u)=\min _{P a} f_{L_{-} P a}\left(P a,-Q^{-}\right) \leq P_{v a r}(u) \leq \max _{P a} f_{L_{-} P a}\left(P a,-Q^{+}\right)=P^{+}(u)
$$

For special case of CCP for networks with active edges $Q^{-}=Q^{+}=0$, and theorem condition are that all P-paths pass zero flow, and inequalities take form

$$
P^{-}(u)=\min _{P a} f_{L_{-} P a}(P a, 0) \leq P_{v a r}(u) \leq \max _{P a} f_{L_{-} P a}(P a, 0)=P^{+}(u)
$$

The proof of theorem 2 can be done by induction on edges number and is constructed practically identical to general existence theorem proof in [1].

Base of induction $\left(N_{E}=1\right)$. For graphs, consisting from 1 edge (and 2 nodes), for the case, when pressure is set in 2 nodes, CFDP is solvable by definition. For the case of set pressure in one node and inflow in another - the edge itself is P-path, and condition on passing $-Q^{+}$and $-Q^{-}$on it provides existence of solution. 
Step of induction. Let graph $G$ has $N_{E}>1$ edges and theorem is proved for any graph with number of edges $<N_{E}$. It is evident, that it needs to be proved only for P-reduced graphs.

So let $G$ - P-reduced graph. Select some node $v^{\prime}$ with set pressure. It has degree 1 and is connect by some edge $e^{\prime}$ with node $v^{\prime \prime} \in V_{Q}$. For further simplification we can change $e^{\prime}$ direction (if necessary) so that it would be directed from $v^{\prime}$ to $v^{\prime \prime}$. Let $G^{\prime \prime}$ - graph, produced from $G$ by deleting $v^{\prime}$ and $e^{\prime}$.

2 cases are possible for $G-N_{P}=1$ и $N_{P}>1$.

In the first case $N_{P}=1$ node $v^{\prime}$ is the only node with set pressures. In this case for CFDP solution, if it exists, we should have $Q_{v a r}\left(v^{\prime}\right)=-\left(Q^{+}+Q^{-}\right)$. Consider P-path $P a^{\prime}$, consisting from edges $e^{\prime}$, and flow value $X^{\prime}=-\left(Q^{+}+Q^{-}\right)$. As $-Q^{+} \leq-\left(Q^{+}+Q^{-}\right) \leq$ $-Q^{-}$and according theorem condition $P a^{\prime}$ passes flows $-Q^{+}$and $-Q^{-}$, it also passes $X^{\prime}$, and $P^{\prime \prime}=P_{v a r}\left(v^{\prime \prime}\right)=f_{L_{-} P a}\left(P a^{\prime}, X^{\prime}\right)$ fits inequalities

$$
P^{-}\left(v^{\prime \prime}\right) \leq f_{L_{-} P a}\left(P a^{\prime},-Q^{-}\right) \leq P^{\prime \prime} \leq f_{L_{-} P a}\left(P a^{\prime},-Q^{+}\right) \leq P^{+}\left(v^{\prime \prime}\right)
$$

Consider CFDP on $G^{\prime \prime}$ with set pressure in node $v^{\prime \prime} P_{\text {fix }}\left(v^{\prime \prime}\right)=P^{\prime \prime}$ the same other source data on $G^{\prime \prime}$, as for original CFDP on $G$. For this CFDP the P-paths passing conditions are fulfilled. Really, comparing these CFDPs: for P-components of $G^{\prime \prime}$ value $Q^{+}$ will not inclease, and $Q^{-}$will not decrease, so P-paths in $G^{\prime \prime}$, starting in any nodes, expect $v^{\prime \prime}$, must pass correspondent flows. And P-paths, starting in $v^{\prime \prime}$, will pass them as parts of P-paths in $G$ (if add to them edge $e^{\prime}$ with flow $X^{\prime}$ ). Thus, according induction assumption CFDP solution on $G^{\prime \prime}$ exists, and nodal pressures for it are between $P^{-}$and $P^{+}$. Along with flow rate $X^{\prime}$ on $e^{\prime}$ it will provide solution of original CFDP on $G$.

Let us now consider the case $N_{P}>1$. Again let's take P-path $P a^{\prime}$, of one edge $e^{\prime}$, and pass zero 0 . As $-Q^{+} \leq 0 \leq-Q^{-}$, and according theorem condition $P a^{\prime}$ passes flows $-Q^{+}$ and $-Q^{-}$, it also passes zero flow, and for such flow $P^{0}=P_{v a r}\left(v^{\prime \prime}\right)=f_{L_{-} P a}\left(P a^{\prime}, 0\right)$ fits

$$
P^{-}\left(v^{\prime \prime}\right) \leq f_{L_{-} P a}\left(P a^{\prime},-Q^{-}\right) \leq P^{0} \leq f_{L_{-} P a}\left(P a^{\prime},-Q^{+}\right) \leq P^{+}\left(v^{\prime \prime}\right)
$$

Let's consider CFDP on $G^{\prime \prime}$ with set pressure in $v^{\prime \prime} P_{f i x}\left(v^{\prime \prime}\right)=P^{0}$ and the same other source data $G^{\prime \prime}$, as for original CFDP on $G$. P-paths passing conditions will be fulfilled for it (for the same reasons, as in case $N_{P}=1$ ). Thus, according induction assuption Solution of such CFDP on $G^{\prime \prime}$ exists, and its nodal pressures $P_{\text {var }}$ are between $P^{-}$and $P^{+}$.

Consider now another CFDP on $G^{\prime \prime}$, with completely the same source data as in original CFDP on $G$ (i.e. with set inflow in $v^{\prime \prime}$ ). It is evident, that for this CFDP P-paths passing conditions are also fulfilled - the values $-Q^{+}$and $-Q^{-}$are the same, but some P-paths (starting in $v^{\prime}$ ) are not necessary to consider. Thus, according induction assumption Solution of this CFDP on $G^{\prime \prime}$ also exists (and $P_{v a r}$ for this solution also are between $P^{-}$and $\left.P^{+}\right)$. Let $P^{*}=P_{v a r}\left(v^{\prime \prime}\right)$. This solution is also a solution of CFDP on $G^{\prime \prime}$ with set pressure $P^{*}$ in $v^{\prime \prime}$.

So we proved, that there are solutions of CFDP on $G^{\prime \prime}$ (and on G) with set pressure in $v^{\prime \prime}$ for 2 pressure value: $P^{0}$ and $P^{*}$ (with the same other source data, and node pressures $P_{v a r}$ between $P^{-}$and $P^{+}$). Then according theorem of intermediate source data [1] solution also exists for any intermediate values of pressure in $v^{\prime \prime}$, and for them $P_{v a r}$ also is between $P^{-}$and $P^{+}$. It remains to find such $P^{\prime \prime} \in\left[P^{0}, P^{*}\right]$ for $v^{\prime \prime}$, for which inflow in $v^{\prime \prime} Q_{v a r}\left(v^{\prime \prime}\right)$ is equal $Q_{f i x}\left(v^{\prime \prime}\right)$ for original CFDP. In this case $Q_{v a r}\left(v^{\prime \prime}\right)=Q_{v a r}^{\prime \prime}\left(v^{\prime \prime}\right)-X^{\prime}$, where $Q_{v a r}^{\prime \prime}\left(v^{\prime \prime}\right)$ - inflow in $G^{\prime \prime}$ (without $e^{\prime}$ ), and $X^{\prime}$ - flow rate on $e^{\prime}$. 
If $P^{0}=P^{*}$, then $P^{\prime \prime}=P^{0}=P^{*}$, as for this pressure value $Q_{v a r}^{\prime \prime}\left(v^{\prime \prime}\right)=Q_{f i x}\left(v^{\prime \prime}\right)$ and $X^{\prime}=0$.

Consider case $P^{0} \neq P^{*}$. According theorem $3[1] Q_{v a r}^{\prime \prime}\left(v^{\prime \prime}\right), X^{\prime}$ and $Q_{v a r}\left(v^{\prime \prime}\right)$ on $\left[P^{0}, P^{*}\right]$ depend on pressure in $v^{\prime \prime}$ continuously, $Q_{v a r}^{\prime \prime}\left(v^{\prime \prime}\right)$ and $Q_{v a r}\left(v^{\prime \prime}\right)$ strictly increase, and $X^{\prime}$ strictly decrease. Let $P^{0}>P^{*}$. The for pressure $P^{0} Q_{v a r}^{\prime \prime}\left(v^{\prime \prime}\right)>Q_{f i x}\left(v^{\prime \prime}\right), X^{\prime}=0$, and for $P^{*} Q_{v a r}^{\prime \prime}\left(v^{\prime \prime}\right)=Q_{f i x}\left(v^{\prime \prime}\right), X^{\prime}>0$. Thus for $P^{0} Q_{v a r}\left(v^{\prime \prime}\right)>Q_{f i x}\left(v^{\prime \prime}\right)$, and for $P^{*}$ $Q_{v a r}\left(v^{\prime \prime}\right)<Q_{f i x}\left(v^{\prime \prime}\right)$. And if $P^{0}<P^{*}$, then similar for $P^{0} Q_{v a r}\left(v^{\prime \prime}\right)<Q_{f i x}\left(v^{\prime \prime}\right)$, and for $P^{*} Q_{v a r}\left(v^{\prime \prime}\right)>Q_{f i x}\left(v^{\prime \prime}\right)$. Thus, function $Q_{v a r}\left(v^{\prime \prime}\right)-Q_{f i x}\left(v^{\prime \prime}\right)$ is continuous, strictly increase and changes sign on $\left[P^{0}, P^{*}\right]$ and must be equal zero in some point $P^{\prime \prime}$ - and this proves the theorem.

Naturally, Theorem 2 conditions are sufficient but not required. They don't take into account network structure and topology, and allow to make just quick and simple estimation. They can be improved (for example by considering trees in $V_{Q}$ ), but this is the topic for separate article.

\section{Abstract hydraulic networks, multiport elements and monotonic properties of CFDP solution}

Results obtained in article [1], can be easily extended to even more general case - if consider them from some another viewpoint (W. C. Rheinboldt [2]). Let list these results.

Suppose that there is some hydraulic (or electric, or any other) network, whose internal structure is unknown. All what is known - that network has $N$ nodes (ports), and when applying some vector of potentials $P$ to them, there will be inflows $Q$ in these nodes. So all what is known, is mapping $\Phi: \Omega^{N} \rightarrow \mathbb{R}^{N}$, linking nodal pressures and nodal inflows: $Q=$ $\Phi(P)$. We will call such networks abstract hydraulic network (AHN). CFDP can be naturally defined for AHN: set pressures $P_{\text {fix }}$ in $N_{P}>0$ nodes (set $V_{P}$ ) and inflows $Q_{\text {fix }}$ in remaining $N_{Q}=N_{V}-N_{P}$ nodes (set $V_{Q}$ ), it is necessary to find pressures $P_{v a r}$ in remaining $N_{Q}$ nodes and inflows $Q_{v a r}$ in $N_{P}$ nodes. Usual networks are also AHN.

It found out that for AHN with function $\Phi$ which has some "natural" properties (similar to describe in [1] for function $\varphi$ ), CFDP solution still has monotonic properties, exists and is unique.

Let $F: \mathbb{R}^{n} \rightarrow \mathbb{R}^{n}$ be some mapping. On the set of vector from $\mathbb{R}^{n}$ partial ordering is defined: $x \leq y$, if for $\forall i x_{i} \leq y_{i} . F$ is called isotonic, if it keeps ordering: $x \leq y \Rightarrow F(x) \leq$ $F(y)$. If, vise verse, it inverts ordering, it is called anti-isotonic (antitonic). $F$ is called diagonally isotonic, if for $\forall i F_{i}(x)$ does not decrease on $x_{i}$ (other variables are fixed). $F$ is call off-diagonal antitonic (ODA), if for $\forall i \neq j F_{i}(x)$ does not increase on $x_{j}$. $F$ is called ballanced, if $\sum_{i=1}^{n} F_{i}=0$. Note, that if $F$ is OFA and balanced, it is diagonally isotonic.

Natural requirements for AHN function $\Phi$, are continuity, balanced and ODA.

AHN behavior is convenient to describe by associated digraphs. Define digraph $\widehat{G}$ in the following way - it has edge from node $v_{i}$ to node $v_{j}(i \neq j)$, only if $P_{i}$ somewhat influences $Q_{j}$ (with fixed other node pressures). In general, this dependence could be not always strictly monotonic (for example, when choked flow occurs), so let define subgraph $\widehat{G}_{\text {Strict }}$, which includes only edges, for which dependence is always strictly decreasing. Finally, define subgraph $\widehat{G}_{\text {Surf }}$, which includes only edges, for which dependence $Q_{j}$ on $P_{i}$ always is not only strictly decreasing, but also surjective (i.e. $Q_{j}$ can have any value of $\mathbb{R}$ when $P_{i}$ 
changes). Connectivity and reachability in graphs $\widehat{G}_{\text {Strict }}$ and $\hat{G}_{\text {Surf }}$ define properties of CFDP solution. The best case is when $\widehat{G}_{\text {Strict }}$ (and $\widehat{G}_{\text {Surf }}$ ) are strongly connected.

Note that connecting (via joint nodes) several AHN with described natural properties, resulting AHN will also have these properties. Thus usual networks have these properties they are connected from edges with such properties. But we can construct AHN not only by 2-port elements - there are such multiport elements in real pipelines as tees, crosses, 3-way and 4-way valves, etc. This approach covers also hydraulic networks with multiport elements - if their behavior follows described natural properties.

Let $\tilde{G}$ - some digraph, and $v_{1}, v_{2}$ - nodes in $\tilde{G}$. We will denote reachability of $v_{2}$ from $v_{1}$ by $v_{1} \underset{\widetilde{G}}{\rightarrow} v_{2}$. Set of all nodes (in $\widetilde{G}$ ) reachable from node $v$, will be denoted $\mathcal{R}_{\tilde{G}}(v)$, and node set, from which $v$ is reachable - by $\mathcal{R}_{\tilde{G}}^{-1}(v)$. For some node subset $S$ of graph $\tilde{G}$, $\mathcal{R}_{\tilde{G}}(S)$ is node set, reachable from $S$, and $\mathcal{R}_{\tilde{G}}^{-1}(S)$ - set of node, from which some node from $S$ is reachable. For $\widehat{G}_{\text {Strict }}$ and $\widehat{G}_{\text {Surf }}$ we will simply use correspondent indexes.

Now we are ready to formulate generalization of result from [1] for AHN.

Lemma $1 \mathrm{~A}$ on pressure change in AHN.

Consider ODA AHN and 2 pressure vectors $P^{(1)}$ and $P^{(2)}$. Consider sets $V^{+}, V^{-}, V^{0}$ :

$v \in V^{+}$when $P^{(1)}(v)<P^{(2)}(v)$

$v \in V^{-}$when $P^{(1)}(v)>P^{(2)}(v)$

$v \in V^{0}$ when $P^{(1)}(v)=P^{(2)}(v)$

Then

1) If $V^{+} \neq V, V^{+} \neq \varnothing$, and $V \backslash V^{+} \cap \mathcal{R}_{\text {Strict }}\left(V^{+}\right) \neq \varnothing$,

then $\sum_{v \in V^{+}} Q^{(1)}(v)<\sum_{v \in V^{+}} Q^{(2)}(v)$

2) If $V^{-} \neq V, V^{-} \neq \varnothing$, and $V \backslash V^{-} \cap \mathcal{R}_{\text {Strict }}\left(V^{-}\right) \neq \varnothing$,

then $\sum_{v \in V^{-}} Q^{(1)}(v)>\sum_{v \in V^{-}} Q^{(2)}(v)$

Theorem 1A (Uniqueness of CFDP solution for AHN)

If AHN is balanced, ODA и $V_{Q} \subset \mathcal{R}_{\text {Strict }}^{-1}\left(V_{P}\right)$, then CFDP solution is unique.

Theorem 2A (Monotonicity of CFDP solution for AHN)

Suppose AHN is balanced, ODA and $V_{Q} \subset \mathcal{R}_{\text {Strict }}^{-1}\left(V_{P}\right)$, and there are 2 CFDP solutions (with the same $V_{P}$ and $V_{Q}$ ), and $P_{f i x}^{(1)} \leq P_{f i x}^{(2)}, Q_{f i x}^{(1)} \leq Q_{f i x}^{(2)}$. Consider $V_{P}^{+}$and $V_{Q}^{+}$subsets of $V_{P}$ and $V_{Q}$, for which inequality is strict. Then:

1) $P_{\text {var }}^{(1)} \leq P_{\text {var }}^{(2)}$, and for node from $\mathcal{R}_{\text {Strict }}\left(V_{P}^{+} \cup V_{Q}^{+}\right) \cap V_{Q} P_{v a r}^{(1)}<P_{v a r}^{(2)}$

2) If $N_{P}>1$, then $Q_{v a r}^{(1)}(v) \geq Q_{v a r}^{(2)}(v)$ on $V_{P} \backslash V_{P}^{+}$, and on $\left(V_{P} \backslash V_{P}^{+}\right) \cap$ $\mathcal{R}_{\text {Strict }}\left(V_{P}^{+} \cup V_{Q}^{+}\right) Q_{\text {var }}^{(1)}(v)>Q_{\text {var }}^{(2)}(v)$

$3)$ If $N_{P}=1$ и $V_{Q}^{+} \neq \varnothing$, then in the only node $v$ with set pressure $Q_{v a r}^{(1)}(v)>$ $Q_{\text {var }}^{(2)}(v)$, otherwise $Q_{v a r}^{(1)}(v)=Q_{v a r}^{(2)}(v)$

4) $\sum_{v \in V_{P}^{+}} Q_{v a r}^{(1)}(v) \leq \sum_{v \in V_{P}^{+}} Q_{v a r}^{(2)}(v)$. If $N_{P}>1, V_{Q}^{+}=\emptyset, V_{P}^{+} \neq \emptyset, V_{P} \backslash V_{P}^{+} \neq \emptyset$, $\left(V_{P} \backslash V_{P}^{+}\right) \cap \mathcal{R}_{\text {Strict }}\left(V_{P}^{+}\right) \neq \emptyset$, then $\sum_{v \in V_{P}^{+}} Q_{v a r}^{(1)}(v)<\sum_{v \in V_{P}^{+}} Q_{v a r}^{(2)}(v)$

Theorem 3A (Continuity and monotonicity of CFDP solution for AHN)

Suppose that AHN is continuous, balanced, ODA and $V_{Q} \subset \mathcal{R}_{\text {Strict }}^{-1}\left(V_{P}\right)$. Then

1. Set $E$, on which CFDP is solvable, is homeomorphic to $\Omega^{N_{V}}$ (or $\mathbb{R}^{N_{V}}$ ) and thus is not empty, open and connected.

2. All solution parameters (nodal pressures and inflows) are continuous functions of source data.

3. The solution has monotonic properties, corresponding to described in theorem $2 \mathrm{~A}$. 
Theorem 4A (AHN CFDP solution existence for intermediate source data).

Suppose that AHN is continuous, balanced, ODA, $V_{Q} \subset \mathcal{R}_{\text {Strict }}^{-1}\left(V_{P}\right)$, and there are 2 CFDP solutions (with the same $V_{P}$ and $V_{Q}$ ), and $P_{f i x}^{(1)} \leq P_{f i x}^{(2)}, Q_{f i x}^{(1)} \leq Q_{f i x}^{(2)}$. Then for any "intermediate" source data $P_{f i x}^{(1)} \leq P_{f i x} \leq P_{f i x}^{(2)}, Q_{f i x}^{(1)} \leq Q_{f i x} \leq Q_{f i x}^{(2)}$ CFDP is also solvable, and $P_{\text {var }}^{(1)} \leq P_{\text {var }} \leq P_{\text {var }}^{(2)}$.

Theorem 5A (CFDP solution existence for AHN)

Suppose that AHN is continuous, balanced, ODA and $V_{Q} \subset \mathcal{R}_{\text {Surf }}^{-1}\left(V_{P}\right)$. Then any CFDP for such AHN is solvable.

We will call AHN passive, if applying any equal pressure to all nodes produces zero inflows.

Tеорема 6A (CCP solution existence for passive AHN).

Let AHN be continuous, balanced, ODA, passive and $V_{Q} \subset \mathcal{R}_{\text {Strict }}^{-1}\left(V_{P}\right)$, and there is CFDP with $Q_{f i x}=0$ and $P_{f i x} \in \Omega^{N_{P}}$. Then CFDP solution exists, and $P_{v a r} \in$ $\left[\min \left(P_{f i x}\right), \max \left(P_{f i x}\right)\right]^{N_{Q}}$.

Proofs of all theorems, except 5A, are just similar to those for usual networks, so are not included in this article. The only interesting proof is for theorem $5 \mathrm{~A}$, which differs from that for Theorem 5 [1], and is more simple than in [2].

The proof uses induction on $N_{V}$ (recursion).

For base case $N_{V}=0$ the Theorem is right by definition.

Suppose that $N_{V}>0$. As $V_{Q} \subset \mathcal{R}_{\text {Surf }}^{-1}\left(V_{P}\right)$, it is always possible to select such node $v^{\prime} \in$ $V_{Q}$, from which some edge $e^{\prime}$ in $\widehat{G}_{\text {Surf }}$ ends in $v^{\prime \prime} \in V_{P}$. Now we will change CFDP source data in $v^{\prime}$ - we will set pressure $P^{\prime}$ in it - and remain other source data the same. Modified CFDP fits theorem conditions and has smaller $N_{V}$, so should have a solution. Let's look how $Q_{v a r}\left(v^{\prime}\right)$ for this solution depends on $P^{\prime}$. From theorems $3 \mathrm{~A}$ and $4 \mathrm{~A}$ we get, that this dependence is continuous and monotonically increasing. From balanced condition $Q_{v a r}\left(v^{\prime}\right)=-\sum_{v \in V_{Q}, v \neq v^{\prime}} Q_{f i x}(v)-\sum_{v \in V_{P}} Q_{v a r}(v)$. The first sum is constant. All terms of the second sum according the same theorems are continuous and not increasing. And there is the term among them, corresponding to node $v^{\prime \prime}$. As there edge from $v^{\prime}$ to $v^{\prime \prime}$ is in $\widehat{G}_{\text {Surf }}$, even if $P_{v a r}(v)$ for nodes $v \in V_{Q}, v \neq v^{\prime}$ would be fixed, $Q_{v a r}\left(v^{\prime \prime}\right)$ would be continuous strictly monotonically decreasing function of $P^{\prime}$ and surjective to $\mathbb{R}$. But really $P_{v a r}(v)$ for $v \in V_{Q}, v \neq v^{\prime}$ continuously increase while $P^{\prime}$ increasing (theorem 3A and 4A) and because of ODA conditions only makes $Q_{v a r}\left(v^{\prime \prime}\right)$ change stronger with $P^{\prime}$ change. So $Q_{v a r}\left(v^{\prime \prime}\right)$ should be surjective on $\mathbb{R}$, and $Q_{v a r}\left(v^{\prime}\right)$ is surjective too. Thus there is some value of $P^{\prime}$, for which $Q_{v a r}\left(v^{\prime}\right)$ is equal to $Q_{f i x}\left(v^{\prime}\right)$ of original CFDP and for which solution of modified CFDP gives the solution of original one.

\section{Sensitivity matrixes and Maxwell matrix}

Now we will return to "usual" hydraulic network (but note that further results can be easily generalized for AHN).

Let suppose, that closure relations are continuously differential in the vicinity of CFDP solution. We will derive sensitivity matrixes (similar to ones obtained in $[5,6,7]$ ), describing CFDP solution sensitivity to source data, and will study how their properties are linked to monotonic properties of CFDP solution. 
Note $d_{F i}=\partial \varphi_{i}\left(P_{F}, P_{L}\right) / \partial P_{F}, \quad d_{L i}=-\partial \varphi_{i}\left(P_{F}, P_{L}\right) / \partial P_{L}$. Then because of $\varphi_{i}$ monotonicity $d_{F i} \geq 0$ and $d_{L i} \geq 0$. Further we will consider only the "regular" case, when $d_{F i}>0$ and $d_{L i}>0$ (and $N_{Q}>0$ ).

Consider diagonal matrixes $D_{F}$ and $D_{L}$ with $d_{F i}$ and $d_{L i}$ on diagonal. Then

$$
d X=\left(D_{F} A_{F}^{T}+D_{L} A_{L}^{T}\right) d P, d Q=A\left(D_{F} A_{F}^{T}+D_{L} A_{L}^{T}\right) d P
$$

Now we will derive Jacobian of mapping $\Psi_{P f i x}$. Remunerate graph nodes so that nodes from $V_{Q}$ come first and split vectors and matrixes to blocks:

$$
P=\left(\begin{array}{c}
P_{v a r} \\
P_{\text {fix }}
\end{array}\right), Q=\left(\begin{array}{c}
Q_{f i x} \\
Q_{v a r}
\end{array}\right), A=\left(\begin{array}{c}
A_{Q} \\
A_{P}
\end{array}\right), A_{F}=\left(\begin{array}{c}
A_{F Q} \\
A_{F P}
\end{array}\right), A_{L}=\left(\begin{array}{c}
A_{L Q} \\
A_{L P}
\end{array}\right)
$$

Then equations (1) gives

$$
\begin{gathered}
\mathrm{dX}=\left(D_{\mathrm{F}} A_{\mathrm{FQ}}^{\mathrm{T}}+\mathrm{D}_{\mathrm{L}} \mathrm{A}_{\mathrm{LQ}}^{\mathrm{T}}\right) d \mathrm{P}_{\mathrm{var}}+\left(\mathrm{D}_{\mathrm{F}} \mathrm{A}_{\mathrm{FP}}^{\mathrm{T}}+\mathrm{D}_{\mathrm{L}} \mathrm{A}_{\mathrm{LP}}^{\mathrm{T}}\right) \mathrm{dP}_{\mathrm{fix}} \\
\mathrm{dQ}_{\mathrm{fix}}=\mathrm{A}_{\mathrm{Q}}\left(\mathrm{D}_{\mathrm{F}} \mathrm{A}_{\mathrm{FQ}}^{\mathrm{T}}+\mathrm{D}_{\mathrm{L}} \mathrm{A}_{\mathrm{LQ}}^{\mathrm{T}}\right) \mathrm{dP}_{\mathrm{var}}+\mathrm{A}_{\mathrm{Q}}\left(\mathrm{D}_{\mathrm{F}} \mathrm{A}_{\mathrm{FP}}^{\mathrm{T}}+\mathrm{D}_{\mathrm{L}} \mathrm{A}_{\mathrm{LP}}^{\mathrm{T}}\right) \mathrm{dP}_{\mathrm{fix}} \\
\mathrm{d} \mathrm{Q}_{\mathrm{var}}=\mathrm{A}_{\mathrm{P}}\left(\mathrm{D}_{\mathrm{F}} \mathrm{A}_{\mathrm{FQ}}^{\mathrm{T}}+\mathrm{D}_{\mathrm{L}} \mathrm{A}_{\mathrm{LQ}}^{\mathrm{T}}\right) \mathrm{dP} \mathrm{P}_{\mathrm{var}}+\mathrm{A}_{\mathrm{P}}\left(\mathrm{D}_{\mathrm{F}} \mathrm{A}_{\mathrm{FP}}^{\mathrm{T}}+\mathrm{D}_{\mathrm{L}} \mathrm{A}_{\mathrm{LP}}^{\mathrm{T}}\right) \mathrm{dP}_{\mathrm{fix}}
\end{gathered}
$$

Matrix $\widetilde{M}=A_{Q}\left(D_{F} A_{F Q}^{T}+D_{L} A_{L Q}^{T}\right)$, which relates $d Q_{f i x}$ and $d P_{v a r}$ (also called modified Maxwell matrix [3, 4]), is Jacobian of $\Psi_{P f i x}$. This matrix has several wonderful properties which will be discussed further. So far just note, that for connected graph and $d_{F i}>0$, $d_{L i}>0$ matrix $\widetilde{M}$ is always nonsingular. So, according implicit function theorem, all CFDP solution parameters are locally continuously differential functions of the source data. Note

$\widetilde{M}_{P P}=A_{P}\left(D_{F} A_{F P}^{T}+D_{L} A_{L P}^{T}\right), \widetilde{M}_{P Q}=A_{P}\left(D_{F} A_{F Q}^{T}+D_{L} A_{L Q}^{T}\right), \widetilde{M}_{Q P}=A_{Q}\left(D_{F} A_{F P}^{T}+D_{L} A_{L P}^{T}\right)(6)$

Then from (4), (5) we can get

$$
\begin{gathered}
\mathrm{dP}_{\mathrm{var}}=\widetilde{\mathrm{M}}^{-1} \mathrm{dQ}_{\mathrm{fix}}-\widetilde{\mathrm{M}}^{-1} \widetilde{\mathrm{M}}_{\mathrm{QP}} \mathrm{dP}_{\mathrm{fix}} \\
\mathrm{dQ} \mathrm{var}_{\mathrm{v}}=\widetilde{\mathrm{M}}_{\mathrm{PQ}} \widetilde{\mathrm{M}}^{-1} \mathrm{dQ}_{\mathrm{fix}}+\left(\widetilde{\mathrm{M}}_{\mathrm{PP}}-\widetilde{\mathrm{M}}_{\mathrm{PQ}} \widetilde{\mathrm{M}}^{-1} \widetilde{\mathrm{M}}_{\mathrm{QP}}\right) \mathrm{dP}_{\mathrm{fix}}
\end{gathered}
$$

Equations (7), (8) give main sensibility matrixes of our problem. For "traditional" networks they coincide with those derived in $[5,6,7]$.

Consider the properties of these matrixes, and first of all - modified Maxwell matrix. The later can be written in the form

$$
\widetilde{M}=A_{Q}\left(D_{F} A_{F Q}^{T}+D_{L} A_{L Q}^{T}\right)=A_{F Q} D_{F} A_{F Q}^{T}+A_{L Q} D_{L} A_{L Q}^{T}+A_{L Q} D_{F} A_{F Q}^{T}+A_{F Q} D_{L} A_{L Q}^{T}
$$

Matrix $A_{F Q} D_{F} A_{F Q}^{T}$ and $A_{L Q} D_{L} A_{L Q}^{T}$ are diagonal. The first one contains on the diagonal sum of $d_{F}$ of all edges starting from corresponding node in i- диагонали, the second one contains the sum of $d_{L}$ for all edges ending in the correspondent node. Matrix $A_{F Q} D_{L} A_{L Q}^{T}$ in line $\mathrm{i}$ and column $\mathrm{j}$ contains sum of $-d_{L}$ for all edges, starting from node $\mathrm{i}$ and ending in node $\mathrm{j}$, while matrix $A_{L Q} D_{F} A_{F Q}^{T}$ contains sum of $-d_{F}$ for all edges, ending in node $\mathrm{i}$ and starting from node j. So matrix $\widetilde{M}$ contains on the diagonal sum of $d_{F}$ for all starting edges plus sum of $d_{L}$ for all ending edges (except edges starting and ending in the same node); non-diagonal cell $m_{i j}$ contains sum of value for all connected nodes $\mathrm{i}$ and $\mathrm{j}$ edges: $-d_{L}$ for edges from $\mathrm{i}$ to $\mathrm{j}$, and $-d_{F}$ for edges from $\mathrm{j}$ to $\mathrm{i}$.

Matrix $\widetilde{M}_{P P}$ has the same structure as $\widetilde{M}$, but for nodes of $V_{P}$. 
For matrixes $\widetilde{M}_{Q P}, \widetilde{M}_{P Q}: \widetilde{M}_{Q P}=A_{F Q} D_{F} A_{F P}^{T}+A_{L Q} D_{L} A_{L P}^{T}+A_{F Q} D_{L} A_{L P}^{T}+A_{L Q} D_{F} A_{F P}^{T}$ and $\widetilde{M}_{P Q}=A_{F P} D_{F} A_{F Q}^{T}+A_{L P} D_{L} A_{L Q}^{T}+A_{F P} D_{L} A_{L Q}^{T}+A_{L P} D_{F} A_{F Q}^{T}$. First 2 terms in both expressions equal 0 , so both matrix contains in ij cell the sum of edge values, connecting nodes $\mathrm{i}$ and $\mathrm{j}:-d_{L}$ for edges from $\mathrm{i}$ to $\mathrm{j}$ and $-d_{F}$ for edges from $\mathrm{j}$ to $\mathrm{i}$. Also $\widetilde{M}_{P Q}=\widetilde{M}_{Q P}^{T}$.

Matrix $\widetilde{M}$ isn't symmetric for the networks with pressure-dependent closure relations (in contrast to "traditional" network). Nevertheless, it keeps her wonderful properties.

First of all, $\widetilde{M}$ is weak diagonally dominant matrix (by columns). Strict diagonal dominance has place for the columns, corresponding nodes, which are connected with nodes from $V_{P}$. As graph is connected and $N_{P}>1$, for any node $V_{Q}$ there is a path to some node from $V_{P}$, so also to some node from $V_{Q}$, connected with $V_{P}$, and edges of such path have correspondent non-zero off-diagonal elements. This makes $\widetilde{M}$ WCDD (Weakly Chained Diagonally Dominant) matrix and non-singular [8].

In the same time $\widetilde{M}$ is also L-matrix: its diagonal elements are positive, while offdiagonal elements are negative or zero. It is already known for a long time ([9]), that matrix, which is both WCDD and L-matrix, is non-singular M-matrix (i.e. matrix with nonpositive off-diagonal elements, with positive real part of its eigenvalues). This class of matrix attracts much attention last years, as it appears in many different fields of mathematics (differential equation, Markov chains etc [10]). The review of M-matrix properties can be found in [11, 12]. Also there are many useful inequalities for their determinant, norm and eigenvalues ([13 - 22]). M-matrixes are also monotonic - their invert matrixes contain only non-negative elements.

Now let study invert Maxwell matrix $\widetilde{M}^{-1}$ in more details.

Non-singular M-matrix $\widetilde{M}$ according Perron-Frobenius theorem can written in the form [10] $\widetilde{M}=s I-B$, where $I-$ identity matrix, and matrix $B$ is non-negative $(B \geq 0)$, while $s$ is more than spectral radius of $B(s>\rho(B))$. The invert matrix can be written as

$$
\widetilde{\mathrm{M}}^{-1}=\mathrm{s}^{-1}\left[\mathrm{I}+\sum_{\mathrm{i}=1}^{\infty} \mathrm{s}^{-\mathrm{i}} \mathrm{B}^{\mathrm{i}}\right]
$$

The row in (9) converges, as $\rho\left(s^{-1} B\right)<1$.

It is evident, that all $\widetilde{M}^{-1}$ elements are non-negative, and all diagonal elements are positive. Which off-diagonal elements are positive? Positive off-diagonal elements of $B$ are elements which are negative in $\widetilde{M}$ - i.e. which corresponds to connected nodes. So in matrix $B^{i}$ all off-diagonal elements, which corresponds to nodes connected by path of I edges with all nodes from $V_{Q}$, are positive (plus maybe some connected with paths of less number of edges). Thus from (9) we get, that positive elements of $\widetilde{M}^{-1}$ are exactly those which corresponds to pair of nodes connected by some path in $V_{Q}$.

Remunerate nodes of $V_{Q}$ grouping them by their P-components. Then matrix $\widetilde{M}$ and $\widetilde{M}^{-1}$ will be block diagonal - each block will correspond to regular (containing nodes from $V_{Q}$ ) P-component. As node from $V_{Q}$ in each P-component form connected subgraph, we have proved the following theorem.

\section{Theorem 3 (On invert Maxwell matrix)}

Modified invert Maxwell matrix $\widetilde{M}^{-1}$ is block diagonal, each block corresponds to regular P-component, and all elements of each block are positive. If graph contains only one P-component, all elements of $\widetilde{M}^{-1}$ are positive.

Now we can see the link between signs of sensitivity matrixes elements and monotonicity of CFDP solution. Theorem 3 corresponds to monotonic increase of $d P_{v a r}$ by $d Q_{f i x}$. Matrix $-\widetilde{M}^{-1} \widetilde{M}_{Q P}$, defining sensitivity of $d P_{v a r}$ to $d P_{f i x}$, also has only positive 
elements for pairs of nodes from the same P-component, and all others equal zero. Matrix $\widetilde{M}_{P Q} \widetilde{M}^{-1}$, defining sensitivity of $d Q_{v a r}$ to $d Q_{f i x}$, has negative elements for nodes in the same P-components, and all others equal zero.

At last, consider matrix $\widetilde{M}_{P P}-\widetilde{M}_{P Q} \widetilde{M}^{-1} \widetilde{M}_{Q P}$, defining sensitivity of $d Q_{v a r}$ to $d P_{f i x}$. According balance equation $d Q_{v a r}$ for $d Q_{f i x}=0$ should also equals 0 . This means that sum of the lines of matrix $\widetilde{M}_{P P}-\widetilde{M}_{P Q} \widetilde{M}^{-1} \widetilde{M}_{Q P}$ equals 0 . In the case when there is only one node with set pressure, all matrix equals 0 . For non-regular P-components diagonal elements are positive, and off diagonal are negative. For regular P-components all offdiagonal elements are negative - so all diagonal elements are positive. This provides known monotonic behavior of $d Q_{v a r}$ on $d P_{f i x}$.

\section{References}

1. L. Korelstein. Proc. MMMAOSDPHS 2019, ID 01004 (2019)

2. Werner C. Rheinboldt, J. of Math. Analysis and applications, 32 (2), 274 (1970)

3. E.A. Mikhailovsky, Piping Systems of Energetics. Mathematical and computer simulation, 34 (Nauka, Novosibirsk, 2014) [in Russian]

4. E.A. Mikhailovsky, N.N. Novitsky, St. Petersburg Polytechnical University Joutnal. Physics and Mathematics, 1 (2), 120 (2015)

5. S.P. Epifanov, N.N. Novitsky, Piping systems of Energetics. Methods of mathematical simulation and optimization, 27 (Nauka, Novosibirsk, 2007) [in Russian]

6. D.I. Borovin, S.P. Epifanov, N.N. Novitsky, Proceeeding of XIV All-Russian Sientific seminar MMMAOSDPHS, 93 [in Russian]

7. S.P. Epifanov, N.N. Novitsky, D.I. Borovin, Piping systems of Energetics. Methodical and applied problems of mathematical simulation, 288 (Nauka, Novosibirsk, 2015) [in Russian]

8. F.R. Gantmaher, The Theory of Matrices, 1(2012); 2 (2018)

9. Parsiad Azimzadeh, https://arxiv.org/pdf/1701.06951.pdf

10. R.J. Plemmons, Linear Algebra and Its Applications, 18, 175 (1977)

11. Charles R. Johnson, Linear Algebra and its Applications, 47, 195 (1982)

12. C. R. Johnson, R. L. Smith, Linear Algebra and its Applications, 435 (5), 953 (2011)

13. Olga Holtz, Proc. of the American Math. Society, 133 (3), 711 (2004)

14. Y.S. Volkov, V.L. Miroshnichenko, Siberian Mathematical Journal, 50 (6), 1248 (2009) [in Russian]

15. Wen Li, Applied Mathematics letters, 21 (3), 258 (2008)

16. Ting-Zhu Huang, Yan Zhu, Linear Algebra and its Applications, 432 (2-3), 670 (2010)

17. Wen Li, Yanmei Chen, J. of Inequalities and Applications, 61 (1), 1 (2012)

18. Gui-Xian Tian, Ting-Zhu Huang, Electronic J. of Linear Algebra, 20 (1), 291 (2010)

19. Ch. Li, Ya. Li, and R. Zhao, Linear and Multiliniar Algebra, 61 (9), 1267 (2013)

20. Feng Wang, Deshu Sun, Open Mathematics, 14 (1), 1107 (2016)

21. J. Zhao, C. Sang, Journal of Inequalities and Applications, Article 119 (2016)

22. Jianxing Zhao, Caili Sang, https://arxiv.org/pdf/1704.05218.pdf 